\title{
Surface roughening during low temperature Si(100) epitaxy
}

\author{
O. P. Karpenko a) and S. M. Yalisove \\ University of Michigan, Department of Materials Science and Engineering, 2300 Hayward Street, \\ Ann Arbor, Michigan 48109-2136 \\ D. J. Eaglesham \\ Bell Laboratories, Lucent Technologies, 600 Mountain Avenue, Murray Hill, New Jersey 07974
}

(Received 19 July 1996; accepted for publication 21 March 1997)

\begin{abstract}
Reflection high energy electron diffraction (RHEED) was used to investigate surface roughening during low temperature $\mathrm{Si}(100)$ homoepitaxy. The use of RHEED allowed in situ real-time collection of structural information from the growth surface. RHEED patterns were analyzed using a simple kinematic diffraction model which related average surface roughness and average in-plane coherence lengths to the lengths and widths of individual RHEED diffraction features, respectively. These RHEED analyses were quantified by calibrating against cross-section transmission electron microscopy (TEM) analyses of surface roughening. Both the RHEED and TEM analyses revealed similar scaling of surface roughness with deposited thickness, with RHEED analyses resulting in roughness values a factor of $\sim 2$ times lower than those obtained from TEM analyses. RHEED was then used to analyze surface roughening during $\mathrm{Si}(100)$ homoepitaxial growth in a range of temperatures, $200-275^{\circ} \mathrm{C}$. Initially, surface roughness increased linearly with deposited thickness at a roughening rate that decreased with increasing growth temperature. At each growth temperature, near the crystalline/amorphous $\mathrm{Si}$ phase transition, the rate of surface roughening decreased. This decrease coincided with the formation of facets and twins along $\mathrm{Si}\{111\}$ planes. Surface roughness eventually saturated at a value which followed an Arrhenius relation with temperature $E_{\text {act }} \sim 0.31 \pm 0.1 \mathrm{eV}$. This activation energy agrees well with the activation energy for the crystalline/amorphous Si phase transition, $E_{\text {act }} \sim 0.35 \mathrm{eV}$, and suggests that limited thickness epitaxy is characterized by this saturation roughness. Once the saturation roughness was reached, no significant changes in surface roughness were detected. In addition, the decay of average in-plane coherence lengths was also temperature dependent. Values of average coherence lengths, at the crystalline/amorphous Si phase transition, also increased with growth temperature. All of these data are consistent with a model that links surface roughening to the formation of critically sized $\operatorname{Si}\{100\}$ facets and the eventual breakdown in crystalline growth. () 1997 American Institute of Physics. [S0021-8979(97)05512-6]
\end{abstract}

\section{INTRODUCTION}

The development of surface roughness during low temperature thin film growth is an important issue with consequences for both the industrial as well as scientific communities. From a technological standpoint, surface roughness is often undesirable and can lead to degraded performance in many applications which utilize thin film heterostructures. For example, the development of surface roughness has a deleterious impact on the operation of various discrete electronic devices (i.e., high electron mobility transistors). ${ }^{1}$ Similarly, as the push for higher density microelectronic circuits continues, the drive to reduce characteristic features sizes within circuit architectures will demand more exacting control over surface and interface roughness within a given heterostructure. As a point of reference, even two monolayers of roughness at the $\mathrm{Si}$ channel/ $/ \mathrm{SiO}_{2}$ gate oxide interface will severely degrade the reliability of this junction due to nonuniformities in the capacitance along the gate. ${ }^{2}$ Indeed, current implementation of chemical mechanical planarization in

\footnotetext{
${ }^{a)}$ Current address: Intel Corporation, 2200 Mission College Blvd. M/S RN235, Santa Clara, CA 95052.
}

semiconductor fabrication highlights industrial concern in reducing roughness between heterolayers within a microelectronic $_{\text {circuit. }}{ }^{3}$

From a scientific standpoint, understanding surface roughening and its effect on the evolution of thin film microstructures is necessary for identification of processes which dominate thin film growth in regimes of limited kinetics. Many groups have already investigated the evolution of surface roughness during growth, etching, and annealing in a number of materials systems. Results from these studies have identified such phenomena as roughness instabilities, ${ }^{4}$ growth of slugs, ${ }^{5}$ and kinetic roughening ${ }^{6}$ where surface roughness follows a power-law dependence on film thickness. ${ }^{7}$ Among the more intriguing materials problems which have been linked to the development of surface roughness is limited thickness epitaxy (LTE) during low temperature semiconductor growth. ${ }^{8-11}$ Past work in the study of low temperature $\mathrm{Si}(100)$ homoepitaxy has shown that the development of surface roughness ${ }^{8}$ and the formation of facets at the growth front ${ }^{9-12}$ are linked to the crystalline/amorphous $\mathrm{Si}$ phase transition. Unfortunately, this phase transition is still not fully understood. One aspect of roughening which requires attention is the scaling of surface roughness with film thickness. The work of Adams et al., looked at surface 
roughening during low temperature $\mathrm{Si}$ homoepitaxial growth prior to the formation of twins and the nucleation of an amorphous Si phase (about 2/3 the critical thickness necessary for the crystalline/amorphous transition). They found that surface roughness follows a power-law dependence on film thickness with a growth exponent $\beta$, greater than $1 .{ }^{8}$ Another study has seen a similar behavior during low temperature $\mathrm{Si}(111)$ homoepitaxy, where surface roughness scaled linearly with film thickness. ${ }^{13}$ In contrast to this behavior, data published in a recent article by Eaglesham show that during amorphous $\mathrm{Si}$ growth, after the crystalline/ amorphous phase transition, surface roughness does not change with film thickness, but rather remains constant. ${ }^{10}$ These two radically different behaviors prompt the question, "Is LTE a critical roughness phenomenon?" This question can only be answered by studying surface roughening of $\mathrm{Si}$ from the start of growth, through the regime of faulted growth, to the crystalline/amorphous phase transition.

This study has focused on developing reflection high energy electron diffraction (RHEED) as a quantitative technique for use in studying surface roughening during low temperature $\mathrm{Si}(100)$ homoepitaxy. RHEED is an ideal tool for this application as it is nonintrusive and provides in situ information about near-surface crystalline structure. Additionally, collection of RHEED data is not hampered by the formation of stacking faults, twins, or other extended defects which hinder transmission electron microscopy (TEM) analyses of surface roughness. ${ }^{14}$ This allows collection of data throughout the entire regime of crystalline growth, from the start of growth, through the region of faulted growth, to the crystalline/amorphous transition. In order to simplify analyses of RHEED patterns, a kinematic model is adopted. ${ }^{6,13}$ Surface roughness in the growth direction was related to the lengths of diffraction features in each RHEED pattern, ${ }^{13,15}$ while, average in-plane coherence lengths were related to the widths of diffraction features in each RHEED pattern. ${ }^{16}$ In order to quantify the RHEED analyses of surface roughness in the growth direction, they were calibrated by direct comparison against TEM analyses of surface roughness. Upon completion of this calibration, RHEED data could easily be interpreted to give accurate measurements of surface roughness without the use of TEM, or the necessity of complicated dynamical diffraction models. ${ }^{17}$ RHEED was then used to analyze surface roughening during $\mathrm{Si}(100)$ homoepitaxy in a range of growth temperatures, $200-275^{\circ} \mathrm{C}$, to determine the scaling of surface roughness with film thickness.

\section{EXPERIMENT}

All Si homoepitaxial growth was performed in a fourchamber cryo-pumped molecular beam epitaxy (MBE) system $\left(P_{\text {base }} \sim 5.0 \mathrm{E}-11\right.$ Torr $)$. Boron-doped, Si(100) wafers, $\rho \sim 1-3 \Omega^{*} \mathrm{~cm}$, were diced to appropriate sizes $(0.25 \mathrm{in}$. $\times 1.20$ in.), degreased, and cleaned using a modified RCA cleaning procedure which left the substrate surfaces capped with a thin volatile oxide layer. ${ }^{18}$ Substrates were mounted onto sample cassettes and introduced into the MBE system via a mechanically pumped loadlock, and moved into a sample preparation chamber. In the sample preparation chamber, each substrate was outgassed for $1.5 \mathrm{~min}$ at $100{ }^{\circ} \mathrm{C}$, $15 \mathrm{~min}$ at $300{ }^{\circ} \mathrm{C}$, and $2 \mathrm{~h}$ at $550^{\circ} \mathrm{C}$. After outgassing, substrates were transferred into the growth and analysis chamber. The protective oxide layer was then thermally volatilized by heating to $\sim 1000{ }^{\circ} \mathrm{C}$ for $2 \mathrm{~min}$. Substrates were cooled to $\sim 400{ }^{\circ} \mathrm{C}$, and a $100 \mathrm{~nm}$ Si buffer layer was grown by electron beam evaporation of a solid Si source. Substrates were subsequently annealed at $\sim 650{ }^{\circ} \mathrm{C}$ while an additional $\sim 50 \mathrm{~nm}$ buffer layer was grown. Following buffer layer growth samples were allowed to equilibrate at the desired growth temperature for $30 \mathrm{~min}$.

RHEED analyses of surface roughness were calibrated against cross-section TEM analyses of surface roughness using the following procedure. Prior to the start of growth, the $\mathrm{Si}$ and $\mathrm{Ge}$ sources were equilibrated at constant deposition rates, $0.03 \mathrm{~nm} / \mathrm{s}$ and $0.005 \mathrm{~nm} / \mathrm{s}$, respectively. Deposition rates were monitored with quartz crystal cantilevers, and were calibrated with Rutherford backscattering spectroscopy and cross-section high resolution (TEM). Each sample was aligned with an electron beam from the RHEED gun ( 25 $\mathrm{keV}, 49 \mu \mathrm{A}$ ), such that the beam was nearly parallel to a $\mathrm{Si}\langle 110\rangle$ zone axis and incident on the surface with a fixed angle between $1^{\circ}$ and $3^{\circ}$ (accurate to within $0.05^{\circ}$ ). RHEED patterns were collected with a charge coupled device camera interfaced to a PC data collection system. ${ }^{19}$ A RHEED pattern was collected prior to any deposition. At the start of growth, a 1/4 monolayer (ML) Ge marker layer was deposited followed by a $15 \mathrm{~nm}$ Si layer. Alternating sequences of 1/4 ML Ge and $15 \mathrm{~nm} \mathrm{Si} \mathrm{continued} \mathrm{to} \mathrm{be} \mathrm{deposited} \mathrm{until} \mathrm{no}$ crystalline features were seen in the RHEED patterns. RHEED patterns were collected and stored for postdeposition analysis after Ge marker deposition and after every $3 \mathrm{~nm}$ of Si deposition. Deposition of $\mathrm{Ge}$ and $\mathrm{Si}$ was controlled by collimation plates and pneumatic shutter mechanisms. $\mathrm{Si}(100)$ homoepitaxial layers without Ge markers were also grown and analyzed with RHEED.

Growth temperatures were calibrated using an optical pyrometer at high temperatures, two low melting point metals (In, $T_{\text {melting }}=430 \mathrm{~K}$ and $\mathrm{Pb}, T_{\text {melting }}=600 \mathrm{~K}$ ) at low temperatures, and current and power interpolation between these data points. Cross-section TEM samples were prepared by substrate cleavage, polishing to $\sim 50 \mu \mathrm{m}$ and ion milling with $5 \mathrm{keV} \mathrm{Ar}{ }^{+}$ions to electron transparency. Cross-section TEM analyses were performed using JOEL 2000 FX analytical electron microscope operating at $200 \mathrm{keV}$.

Values of average surface roughness $\omega_{\text {ave }}$ were obtained from TEM analyses by measuring the spreading in the Ge marker layers from cross-section TEM micrographs. Micrographs were taken from foils $\sim 50 \mathrm{~nm}$ thick, using a kinematic symmetric three-beam, $g=400$, bright field imaging condition. ${ }^{20}$ These micrographs were scanned into a computer using a digital scanner. Following scanning, the intensity profiles of the Ge marker layers were fitted with Gaussians to determine their full widths at half-maximum (FWHM). The FWHM values were then converted to length scales using an appropriate conversion factor.

Values of average in-plane coherence lengths $L_{x}$ and average surface roughness in the direction of growth $\omega_{\text {ave }}$ were determined from the RHEED patterns using a kinematic dif- 
fraction model. ${ }^{6,13}$ All of the primary diffraction maxima in each RHEED pattern were fitted with Gaussian profiles both across the diffraction features (parallel to the plane of growth) and along the diffraction features (normal to the plane of growth). Values of $L_{x}$, in the direction perpendicular to the electron beam direction, were calculated from the FWHM across the diffraction features, ${ }^{16}$ using the following equation,

$$
L_{x}=\frac{\lambda_{e}}{\left(\delta \phi-\delta \phi_{\text {beam }}\right)},
$$

where $\lambda_{e}$ is the electron wavelength, $\delta \phi$ is the FWHM, in milliradians, across the RHEED streak/spot, $\delta \phi_{\text {beam }}$ is the FWHM of the main electron beam in milliradians, and a $\cos \theta_{i}$ term was omitted in the denominator ( $\theta_{i}$ the incident angle of the electron beam is very small, so $\left.\cos \theta_{i}=1\right)$. It is assumed that the measured FWHM of the RHEED streak/ spot is a convolution of instrument broadening and broadening due to the reciprocal lattice rods/spots. Values of $\omega_{\text {ave }}$ were calculated from the FWHM along the diffraction features, using the following equation,

$$
\omega_{\mathrm{ave}}=\frac{\lambda_{e}}{\delta \vartheta},
$$

where $\delta \vartheta$ is the FWHM, in milliradians, along the RHEED streak/spot. Limitations to the approach are discussed in Sec. IV.

\section{RESULTS}

RHEED analyses of surface roughness were compared to TEM analyses of surface roughness in order to determine the quantitative relationship between RHEED and TEM, and to obtain a calibration factor to relate the two techniques. This comparison was conducted at several temperatures (200, 225, 250, and $275^{\circ} \mathrm{C}$ ). Figures 1(a) and 2(a) show typical cross-section TEM micrographs of Si homoepitaxial growth at $T \sim 250{ }^{\circ} \mathrm{C}$ and $T \sim 275{ }^{\circ} \mathrm{C}$. Figures 1(b) and 2(b) show a series of RHEED patterns, along $\mathrm{Si}\langle 011\rangle$, collected during the growth of the films shown in Figs. 1(a) and 2(a), respectively. Both sets of figures show a noticeable increase in $\omega_{\text {ave }}$ with film thickness; spreading in the Ge marker layers (TEM), and a transition from streaked to spotty diffraction patterns (RHEED). The increase in $\omega_{\text {ave }}$ with film thickness for growth at $T \sim 250{ }^{\circ} \mathrm{C}$ and $T \sim 275^{\circ} \mathrm{C}$ is summarized in Figs. 1(c) and 2(c), respectively. Both data sets indicate that $\omega_{\text {ave }}$ increases almost linearly with film thickness. However, the RHEED values of surface roughness are lower than the TEM values by a factor of $\sim 2$. In total, ten films were analyzed for this comparison.

RHEED analyses of $\omega_{\text {ave }}$ were also tested over a range of incident angles $\left(1^{\circ}-3^{\circ}\right.$, inclusive $)$ of the RHEED electron beam. RHEED data were collected from samples grown at $T \sim 250{ }^{\circ} \mathrm{C}$ with the incident angle of the electron beam set at a fixed angle for the entire experiment. The incident angles investigated included $1.0^{\circ}, 2.0^{\circ}, 2.5^{\circ}$, and $3.0^{\circ}$. Two samples were grown at each incident angle to determine the influence of diffraction geometry on RHEED analyses. The result of these experiments suggest that RHEED analyses are insensi- tive to changes in the incident electron beam angle over the range of angles tested, as shown in the plot in Fig. 3.

After calibration of the kinematic RHEED model, additional experiments were conducted where only RHEED was used to monitor the development of surface roughness during $\mathrm{Si}(100)$ homoepitaxy. The growth temperatures tested in these experiments included $200,225,250$, and $275^{\circ} \mathrm{C}$. The evolution of $\omega_{\text {ave }}$ as a function of deposited thickness is shown in Fig. 4. This plot shows an initially linear increase in $\omega_{\text {ave }}$ with deposited thickness, where the rate of surface roughening decreases with increasing growth temperature. At each growth temperature, the rate of surface roughening as a function of deposited thickness eventually decreased and saturated at a value $\omega_{\text {sat }}$ near the crystalline/amorphous $\mathrm{Si}$ phase transition. The value of $\omega_{\text {sat }}$ followed an Arrhenius relation with growth temperature and was characterized by an activation energy, $E_{\text {act }} \sim 0.31 \pm 0.1 \mathrm{eV}$ (see Fig. 5). At a given growth temperature, once $\omega_{\text {sat }}$ was reached, the measured surface roughness remained constant until continued growth was entirely amorphous (typically $\sim 20-40 \mathrm{~nm}$ after $\omega_{\text {sat }}$ was reached). In addition, the point at which the rate of surface roughening with deposited thickness decreased correlated to the onset of faceting and twinning in the crystalline layer, as indicated by the formation of additional diffraction maxima in the RHEED patterns (see Fig. 6).

The evolution of average in-plane coherence lengths $L_{x}$ as a function of deposited thickness was also investigated. These results are summarized in the plot in Fig. 7. For growth at all temperatures, $L_{x}$ eventually decays as a function of deposited thickness. This decay occurs almost immediately for growth at 200 and $225^{\circ} \mathrm{C}$. For growth at 250 and $275^{\circ} \mathrm{C}, L_{x}$ increases initially, and then starts to decay after deposition of some temperature dependent layer thickness ( $\sim 30 \mathrm{~nm}$ for growth at $250{ }^{\circ} \mathrm{C}$ and $\sim 100 \mathrm{~nm}$ for growth at $275^{\circ} \mathrm{C}$ ). In addition, $L_{x}$ at the crystalline/amorphous transition increased with growth temperature.

\section{DISCUSSION}

\section{A. RHEED calibration}

Prior to discussing surface roughening during $\mathrm{Si}(100)$ homoepitaxy, it is important to address the limitations of the kinematic approach used to interpret RHEED data. The main reason for choosing a kinematic interpretation of the RHEED data is simplicity. As mentioned above, both in-plane coherence lengths and surface roughness can be related to the FWHM across and along individual RHEED streaks using Eqs. (1) and (2), respectively. No consideration is given to complicated inelastic electron/solid interactions or to multiple scattering effects. ${ }^{17}$ However, the use of this analysis relies on several restrictive approximations, as mentioned in Ref. 13. In order to address these restrictions, it is necessary to consider factors which affect the size and shape of RHEED diffraction features. Since RHEED patterns are simply the intersection of the Ewald sphere with reciprocal space, these factors can be reduced to changes in reciprocal space and to the intersection of reciprocal space with the Ewald sphere. 

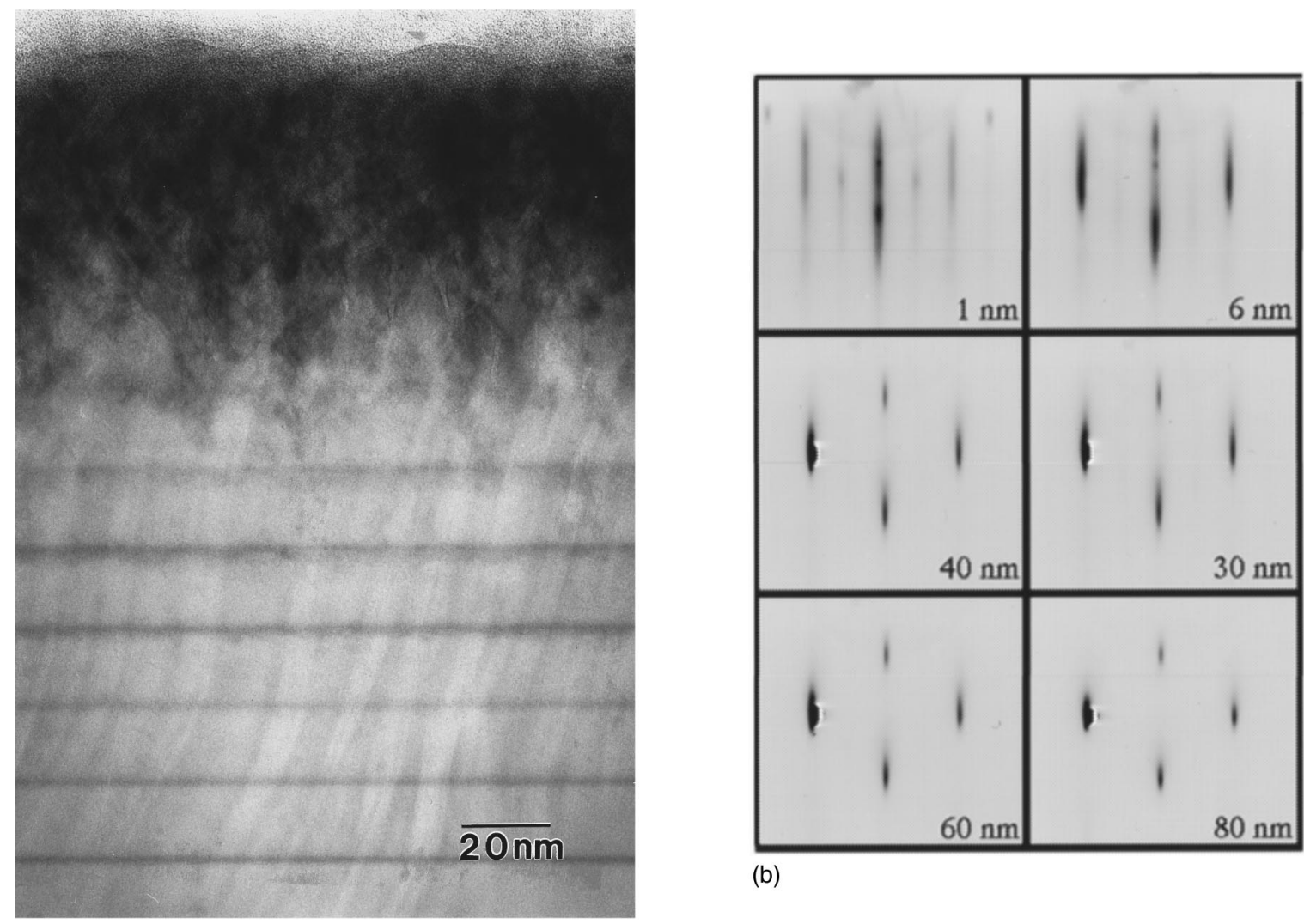

(b)

(a)

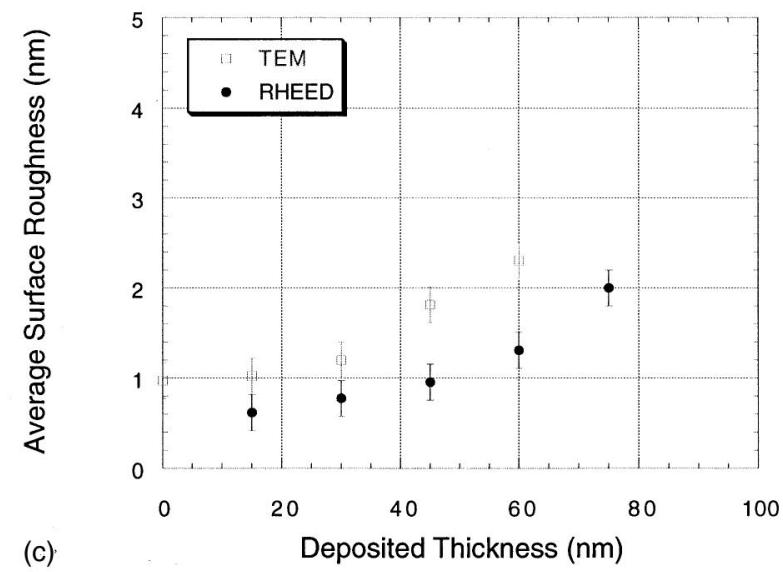

FIG. 1. (a) Bright field, cross-section TEM micrograph of a $\mathrm{Si}(100)$ homoepitaxial layer grown at $T \sim 250{ }^{\circ} \mathrm{C} .1 / 4$ ML Ge marker layers were introduced at the start of growth and after $15 \mathrm{~nm}$ growth intervals of Si. The micrograph was taken using a symmetric three-beam condition, $\mathbf{g}=040$. (b) A series of RHEED patterns collected during growth of the $\mathrm{Si}(100)$ homoepitaxial layer shown in Fig. 1 (a), $T \sim 250^{\circ} \mathrm{C}$. The RHEED beam $(25 \mathrm{keV}, 49 \mu \mathrm{A})$ was aligned nominally along a $\mathrm{Si}\langle 110\rangle$ direction at an incidence angle of $1^{\circ}$. (c) Results of the RHEED and TEM analyses of surface roughness as a function of deposited film thickness for the layer shown in Fig. 1(a).

First, consider the reciprocal space associated with a flat well ordered surface. Due to the limited penetration of electrons normal to the surface in a typical RHEED geometry, the Bragg condition is relaxed in this direction. The result is a reciprocal space consisting of long weakly modulated reciprocal lattice rods normal to the surface, with the reciprocal lattice rod length being related to surface roughness. ${ }^{13,15} \mathrm{Be}-$ cause the Bragg conditions are not relaxed for directions parallel to the surface, the diameter of these rods should be related to the crystalline quality near the surface. ${ }^{15,16,21}$ As this surface becomes rough, the reciprocal lattice rods will shorten in length and condense into Bragg spots of bulk Si. In addition, the width of these Bragg spots will increase to reflect any deterioration in crystalline quality near the surface. The series of RHEED patterns in Figs. 1(a) and 2(a) illustrate this transition from a flat surface (long thin diffraction streaks) to a rough surface (short wide diffraction spots).

It is also important to consider the intersection of the Ewald sphere with reciprocal space. If the Ewald sphere forms an intersection along the entire length of a Bragg spot/ rod, then the length of the resultant diffraction feature will be related to surface roughness. This condition is most easily 

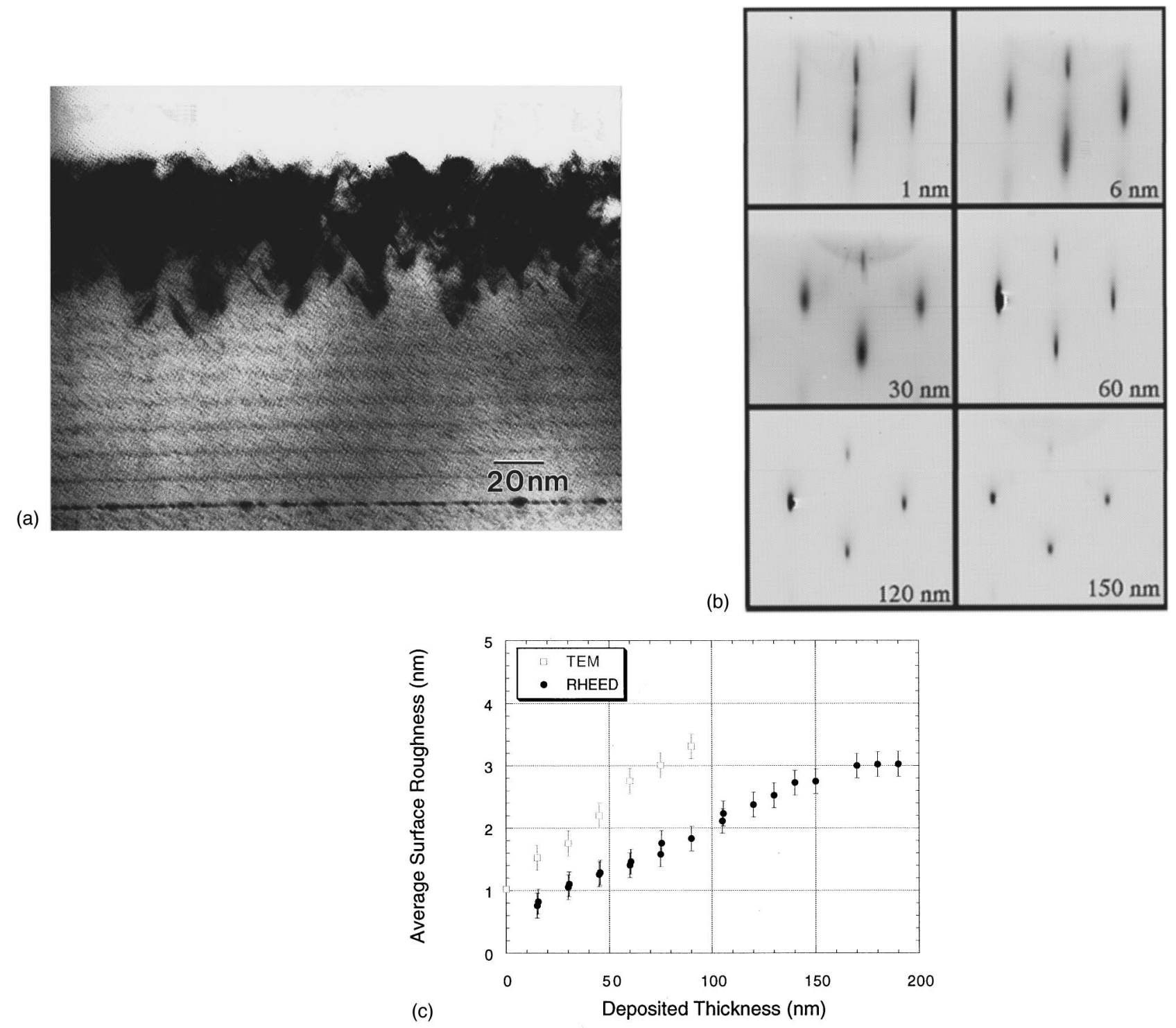

FIG. 2. (a) Bright field, cross-section TEM micrograph of a $\mathrm{Si}(100)$ homoepitaxial layer grown at $T \sim 275^{\circ} \mathrm{C}$. See Fig. 1(a) caption for additional details. (b) A series of RHEED patterns collected during growth of the $\mathrm{Si}(100)$ homoepitaxial layer shown in Fig. $2(\mathrm{a}), T \sim 27{ }^{\circ} \mathrm{C}$. See Fig. 2 (a) for additional details. (c) Results of the RHEED and TEM analyses of surface roughness as a function of deposited thickness for the layer shown in Fig. 2(a).

satisfied in the zeroeth order Laue zone, where the reciprocal lattice spots/rods are nearly tangent to the edge of the Ewald sphere. However, one can imagine that for very flat surfaces, the length of the reciprocal lattice rods will exceed their intersection length with the Ewald sphere. In this case, the length of the RHEED streak will be dominated by instrumental resolution (Ewald sphere radius/electron wavelength) as opposed to surface morphology. This imposes a lower limit on resolvable surface roughness. It is also important to realize that the length of the RHEED diffraction maxima depends on both the length and the width of the reciprocal lattice rods/spots. ${ }^{16}$ For RHEED streaks which are much longer than they are wide, the contribution of reciprocal rod/ spot broadening to the length of the diffraction maxima can be ignored. However, as the RHEED spot length approaches the spot width [see Fig. 2(b)], this approximation is no longer valid, and information about surface roughness can no longer be deconvoluted from the RHEED pattern. This imposes an upper limit on resolvable surface roughness.

The determination of a quantitative relationship between the kinematic RHEED analyses and cross-section TEM analyses of surface roughness $\omega_{\text {ave }}$ allows the extraction of quantitative information from RHEED data using the simple kinematic approach. The primary difference between the two data sets is a scale factor, with RHEED analyses giving values of $\omega_{\text {ave }} \sim 2$ times smaller than TEM analyses. The origin of this discrepancy is a secondary issue, as RHEED values of $\omega_{\text {ave }}$ can be multiplied by a calibration factor to give good agreement with the TEM measurements of surface roughness. However, for the sake of completeness, two possible reasons for the difference between RHEED and TEM values of surface roughness are mentioned. One explanation for differences in these measurements could be related to the different length scales over which RHEED and TEM measure 


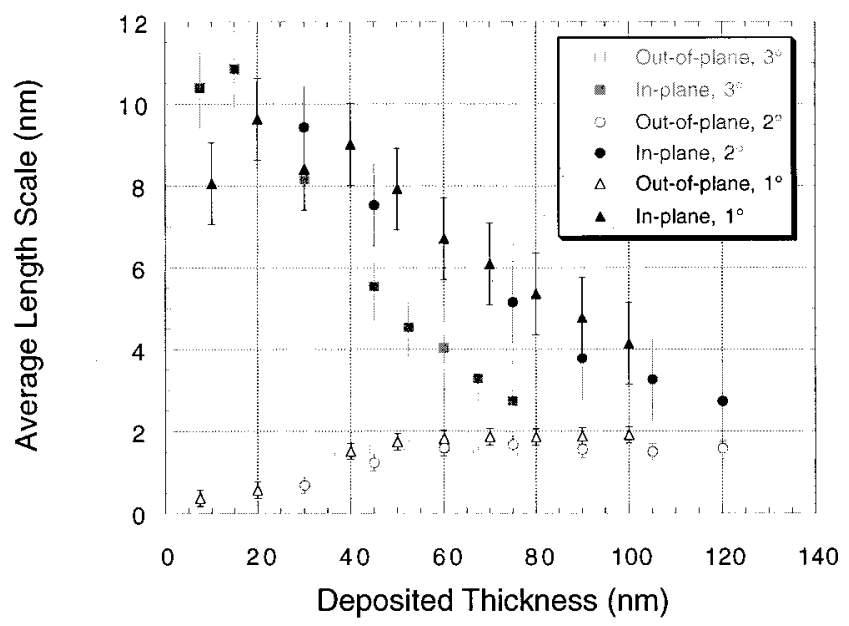

FIG. 3. A plot showing the influence of changing the incident electron beam angle on RHEED analyses of surface roughness. RHEED patterns were collected during growth of $\mathrm{Si}(100)$ homoepitaxial layers at $T \sim 250^{\circ} \mathrm{C}$. The RHEED beam was aligned nominally along a $\operatorname{Si}\langle 110\rangle$ direction.

surface roughness. Since neither the TEM nor the RHEED data were normalized to the length scales over which they probe surface roughness, proper division of these data by appropriate length scales could reduce the difference in the values of surface roughness obtained from these techniques. However, this is unlikely because typical RHEED length scales, $\sim 100 \mathrm{~nm}$, are comparable if not larger than typical TEM length scales, $\sim 50 \mathrm{~nm}$.

A more likely explanation for the difference in measured $\omega_{\text {ave }}$ could be related to the sensitivity of each technique to different geometries of surface roughness. TEM analyses should be sensitive to nearly all geometries of surface roughness, as TEM maps the distribution of Ge along the growth front or a buried interface. RHEED, on the other hand, relies on transmission or reflection of electrons through or from asperities. Due to limited penetration of electrons at the surface, RHEED may underestimate the roughness of surfaces which contain asymmetric asperities, or asperities with large lateral length scales. An example of such a surface is shown

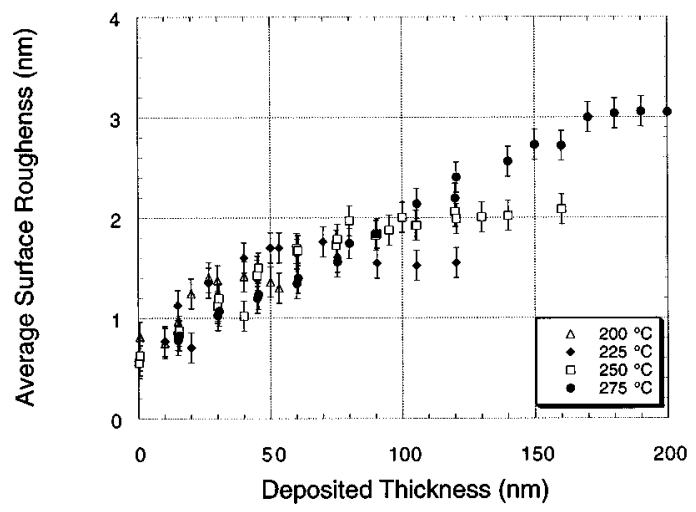

FIG. 4. RHEED analyses of average surface roughness as a function of deposited thickness for $\mathrm{Si}(100)$ homoepitaxial growth at $T \sim 200,225,250$, and $275^{\circ} \mathrm{C}$. RHEED patterns were collected during growth with the RHEED beam aligned along a $\operatorname{Si}\langle 110\rangle$ direction at an incident angle of $1.0^{\circ}$.

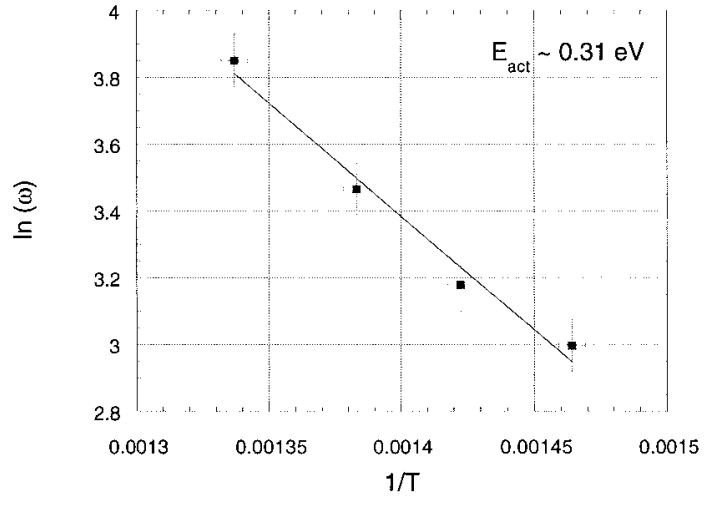

FIG. 5. A plot showing the temperature dependent Arrhenius behavior of the critical saturation roughness. A fit to these data suggests an activation energy of $\sim 0.31 \pm 0.1 \mathrm{eV}$, as compared to an activation energy of $\sim 0.35 \mathrm{eV}$ for the formation of an amorphous Si phase.

in Fig. 8; a micrograph of $\mathrm{Si}(100)$ homoepitaxial growth at $T \sim 350{ }^{\circ} \mathrm{C}$. The surface contains large faceted asperities separated by deep void tracts. This surface geometry might limit electron penetration to the top fraction of each asperity and could lead to the underestimation of surface roughness. Since TEM probes the concentration profile of Ge along an interface, TEM analyses of this surface would more likely reflect the distribution of heights along the $\mathrm{Si}$ surface and should give a higher estimate of $\omega_{\text {ave }}$.

The primary reason for testing the dependence of RHEED analyses on incident electron beam angle was to determine possible errors associated with electron beam drift. Beam drifts as large as $0.25^{\circ}$ have been encountered in our MBE system during growth experiments, although typical excursions are less than $0.1^{\circ}$. The insensitivity of RHEED analyses to changes in the incident angle of the electron beam, over a range of angles $1^{\circ}-3^{\circ}$, suggests that beam drift does not have a significant impact on the results of the RHEED analyses used here.

\section{B. Analysis of surface roughening}

The RHEED analyses display an initially linear dependence of average surface roughness $\omega_{\text {ave }}$ on deposited thick-

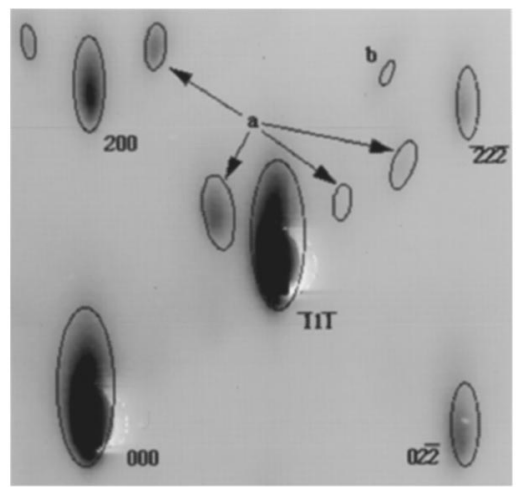

FIG. 6. RHEED pattern collected after $\sim 160 \mathrm{~nm}$ of Si deposition during growth of a $\mathrm{Si}(100)$ homoepitaxial film at $T \sim 275^{\circ} \mathrm{C}$. The diffraction spots labeled (a) correspond to $\mathrm{Si}\{111\}$ facets and twins along $\mathrm{Si}\{111\}$ planes. The diffraction spots labeled (b) correspond to $\mathrm{Si}\{311\}$ facets. 


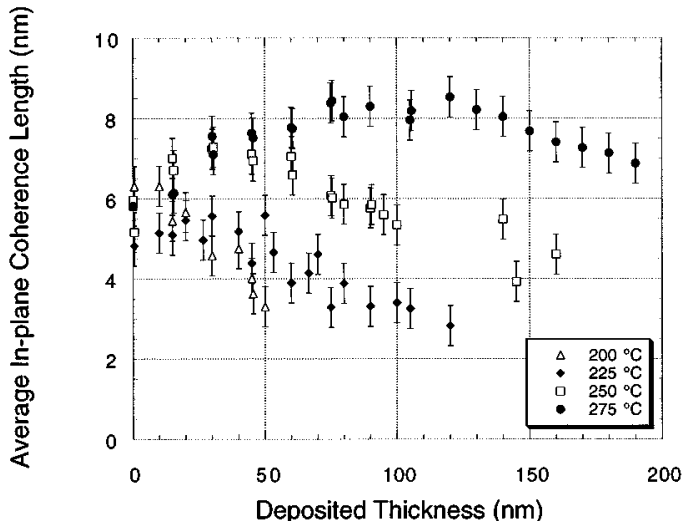

FIG. 7. A plot showing the variation in in-plane coherence length as a function of deposited thickness and growth temperature. All in-plane coherence lengths were calculated from RHEED patterns collected during $\mathrm{Si}(100)$ homoepitaxial growth. The RHEED beam was aligned along a $\mathrm{Si}\langle 110\rangle$ direction at an incident angle of $1.0^{\circ}$.

ness, and a roughening rate that decreases with increasing temperature. A near linear dependence of average surface roughness on deposited thickness has also been reported in at least two other independent studies. ${ }^{8,13}$ All of these results disagree with the predictions of various kinetic roughening models which suggest that surface roughness should follow a power-law dependence on layer thickness with a growth exponent $\beta$ between 0.25 and $0.5 .^{7}$ Possible reasons for this discrepancy are likely related to improper or over simplified treatment of mass transport and adatom diffusion on the $\mathrm{Si}$ surface in these models.

The temperature dependent roughening rate seen in this study could be influenced by a number of factors which affect surface diffusion of Si adatoms. These factors could include but are not limited to an Ehrlich barrier, an energy barrier to adatom diffusion across a step edge, ${ }^{2,23}$ and impurities on the Si surface. ${ }^{24,25}$ For example, recent work by Tersoff et al. has explored the effect of an Ehrlich barrier on the critical island size and the nucleation rate for the formation of a new layer on an island. ${ }^{23}$ Their treatment of the problem suggests that the decrease in nucleation rate for a second layer atop an island with increasing temperature is accelerated by step edge barriers to diffusion. Additionally, the increase in critical island size with increasing temperature, for nucleation of a second layer, is accelerated in the presence of the Ehrlich barrier. For a system which exhibits multilayered growth [clearly the case for low temperature $\mathrm{Si}(100)$ homoepitaxy], both of these trends would lead to decreased rates of surface roughening and increased average in-plane coherence lengths with increasing growth temperature; behaviors which are observed in this study. Similar trends could also be expected in the absence of a step edge barrier to adatom diffusion and for a number of other reasons.

The presence of $\mathrm{H}$ would also affect surface diffusion and surface roughening during low temperature $\mathrm{Si}$ homoepitaxy. ${ }^{8,26}$ Recent studies suggest that $\mathrm{H}$ inhibits $\mathrm{Si}$ surface diffusion via several mechanisms including site-blocking ${ }^{24,25}$ and adatom trapping. ${ }^{25}$ These mechanisms

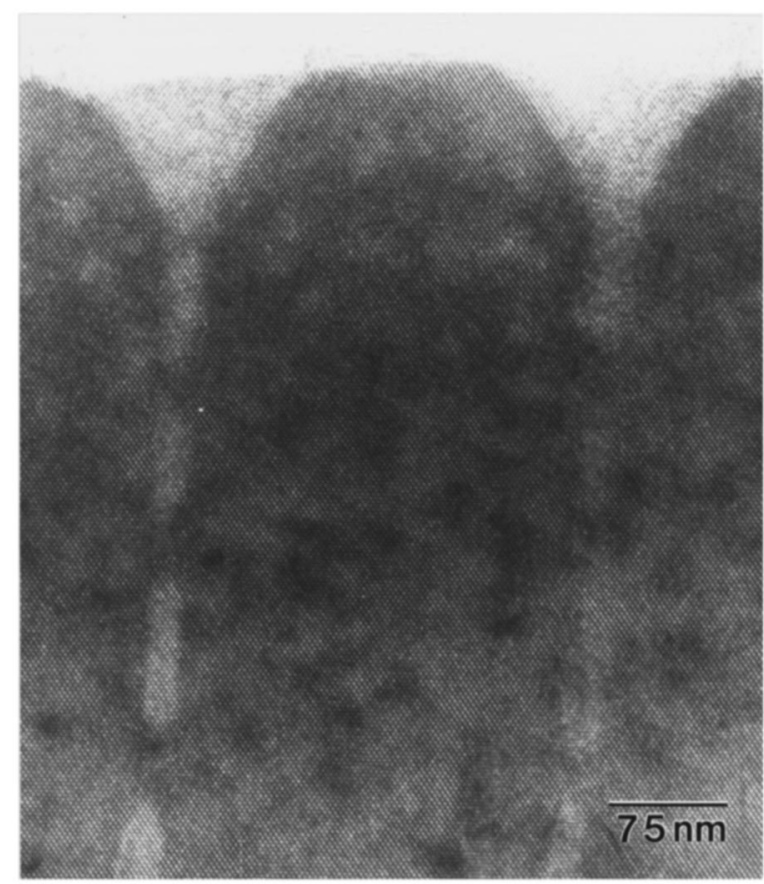

FIG. 8. High resolution cross-section TEM micrograph, along a $\mathrm{Si}\langle 110\rangle$ zone axis, of a $\mathrm{Si}(100)$ homoepitaxial layer grown at $T \sim 350^{\circ} \mathrm{C}$.

manifest themselves in an increased effective activation energy for Si surface diffusion, ${ }^{25}$ and act to accelerate the rate of surface roughening during growth. ${ }^{8} \mathrm{H}$ has also been shown to increase the island nucleation density at the initial stages of low temperature $\mathrm{Si}(100)$ homoepitaxial growth, ${ }^{25}$ as compared to growth in "clean" UHV conditions. In addition, $\mathrm{H}$ can modify surface diffusion processes by tying off bonds and "breaking" surface reconstructions. ${ }^{10,26}$ Other aspects concerning the behavior of $\mathrm{H}$ on $\mathrm{Si}$ surfaces and its effect on low temperature homoepitaxy (i.e., $\mathrm{H}$ mobility on a surface, ${ }^{25}$ effect of various species of $\mathrm{H}^{27}$ ) could also affect surface roughening.

Perhaps the most intriguing result of the RHEED roughening data is the existence of a "saturation" roughness $\omega_{\text {sat }}$. At all growth temperatures, after the initial linear increase in $\omega_{\text {ave }}$ further roughening occurs at a slower rate and eventually saturates at a constant value near the crystalline/ amorphous Si phase transition. For the range of temperatures examined in this study, the value of $\omega_{\text {sat }}$ followed an Arrhenius relation with temperature and was characterized by an activation energy, $E_{\text {act }} \sim 0.31 \mathrm{eV} \pm 0.1 \mathrm{eV}$ (see Fig. 5), in good agreement with the activation energy for LTE on $\mathrm{Si}(100)$ in our MBE systems, $E_{\text {act }} \sim 0.35 \mathrm{eV} .{ }^{9}$ The close agreement in activation energies suggests that the measured $\omega_{\text {sat }}$ may be a critical length scale associated LTE for $\mathrm{Si}(100)$ homoepitaxy. Typically, $\omega_{\text {sat }}$ occurred at $\sim 20-40 \mathrm{~nm}$ prior to the loss of all crystalline diffraction features in the RHEED patterns (the fraction of amorphous material at a given deposited thickness is one). Since the epitaxial thickness $h_{\text {epi }}$ is defined as the deposited thickness where the fraction of crystalline material is $1 / 2$, the thickness when $\omega_{\text {sat }}$ is reached lies somewhere between the point where amorphous Si first starts to form and $h_{\text {epi }}$. The question which remains 
to be answered concerns the physical significance of $\omega_{\text {sat }}$.

One interpretation for $\omega_{\text {sat }}$ is that $\omega_{\text {sat }}$ is the critical roughness necessary to form an amorphous $\mathrm{Si}$ phase. This interpretation seems unlikely. It does not provide a clear mechanism for the formation of an amorphous Si phase. Furthermore this interpretation does not address the formation of $\mathrm{Si}\{111\}$ facets prior to $h_{\text {epi }}$, nor does it explain the scaling of average in-plane coherence lengths with film thickness and growth temperature. Another interpretation for $\omega_{\text {sat }}$ is that it is an artifact of the RHEED measurement technique (see the discussion above). Careful analyses of the RHEED data show that the measurement of $\omega_{\text {sat }}$ is not an artifact, but a value representative of the average surface roughness at $h_{\text {epi }}$.

The best interpretation of the RHEED and TEM data, is that $\omega_{\text {sat }}$ is related to a critical $\operatorname{Si}\{111\}$ facet size necessary for the formation of the amorphous $\mathrm{Si}$ phase. Inspection of RHEED data shows that facets and twins form before $\omega_{\text {ave }}$ reaches its saturation value. The presence of twins and $\mathrm{Si}\{111\}$ facets was identified by weak 1/3 111 type diffraction maxima in the RHEED patterns, while the presence of other $\mathrm{Si}$ facets was identified by additional diffraction maxima (see Fig. 6). Furthermore, inspection of TEM micrographs, when compared to the RHEED data, also suggests that $\omega_{\text {sat }}$ is reached after the formation of twins and facets along the Si surface, and that twin and facet sizes seen in the film are of the same length scale as $\omega_{\text {sat }}$. In addition, the in-plane coherence length data, shown in Fig. 7, also shows that $L_{x}$ decays to a value, at $h_{\text {epi }}$, which increases with growth temperature. This coexistence of large average inplane coherence lengths and large average surface roughnesses at $h_{\text {epi }}$ (e.g., growth at $275^{\circ} \mathrm{C}$ ) is indicative of large facets at the growth front; large average in-plane coherence lengths suggest well-ordered features along the growth front (see also Fig. 6).

Based on the data presented in this study, a model is proposed which links surface roughening to the formation of critically sized $\mathrm{Si}\{111\}$ facets and the eventual breakdown in crystalline growth. The key features of this model include; (1) the existence of temperature dependent critical length scales for LTE, $\omega_{\text {sat }}\left(E_{\text {act }} \sim 0.31 \mathrm{eV}\right)$ and $L_{x}$, (2) the formation of facets at the growth surface prior to $h_{\mathrm{epi}}$, and (3) the formation of amorphous $\mathrm{Si}$ on $\mathrm{Si}(111)$ during low temperature homoepitaxy. ${ }^{28}$ Due to various diffusion barriers along the growth surface, growth at low temperatures leads to rapid surface roughening. The development of surface roughness eventually leads to the formation of $\mathrm{Si}\{111\}$ facets along the growth front. This may be driven by a reduction in total surface energy; the formation of well ordered low energy facets from rough higher energy islanded surfaces. ${ }^{29}$ As growth continues, these facets also grow until they reach a critical size which is characterized by $\omega_{\text {sat }}$ and $L_{x}$. Once the $\mathrm{Si}\{111\}$ facets reach their critical size, a size which is related to the mean adatom diffusion length along the surface, adatoms can no longer diffuse off the $\operatorname{Si}\{111\}$ surfaces. At this point, continued growth along the critically sized $\mathrm{Si}\{111\}$ facets will be dominated by $\mathrm{Si}(111)$ growth mechanisms. This will lead to the formation of twins and stacking faults, and the eventual "nucleation", of an amorphous Si phase. ${ }^{28}$
Additionally, the presence of impurities during growth could affect the critical facet size necessary for LTE by modifying surface diffusion and growth mechanisms along $\mathrm{Si}\{111\}$.

It is speculated that the formation of an amorphous $\mathrm{Si}$ phase first occurs at the intersection of defects at the growth surface. Previous models for LTE have suggested that amorphous Si first forms at the pyramidal intersection of four $\mathrm{Si}$ facets for growth on $\mathrm{Si}(100) .{ }^{10}$ However, this behavior has not been generally observed for growth on $\mathrm{Si}(111)$. Instead, the amorphous-crystalline interface is irregular and is characterized by a high defect density in the crystalline region. Due to the complexities of growth on $\mathrm{Si}(111)$ surfaces, twins easily form along $\{111\}$ planes, ${ }^{28}$ as do other defects. ${ }^{13}$ These defects have been linked to the formation of amorphous Si. The intersection of defects along the Si surface may provide localized regions which are highly disordered and which favor the formation of an amorphous Si phase. In this context, the intersection of facets which contain defected Si layers may also serve as nucleation sites for amorphous $\mathrm{Si}$.

Because it is difficult to calibrate absolute growth temperature, direct quantitative comparisons between LTE results reported by different groups (i.e., growth in different deposition systems) is problematic. For example, the values for the activation energy associated with $\mathrm{Si}(100) \mathrm{LTE}$ have been reported as $0.35 \mathrm{eV}$ by Adams et al. and $0.45 \mathrm{eV}$ by Eaglesham et al. In light of this consideration, the LTE results reported by Adams (see Ref. 9) are most applicable to the results reported here (i.e., our experiments were performed in the same growth system). Adams reports a very rapid transition to amorphous growth on $\mathrm{Si}(111)$, for growth temperatures between 200 and $300{ }^{\circ} \mathrm{C}$, and a much slower transition to amorphous growth on $\mathrm{Si}(100)$. Since these results were obtained from analyses of growth on patterned $\mathrm{Si}(100)$ wafers, (i.e., on a number of different $\mathrm{Si}\{h k l\}$ surfaces), the relative temperatures for growth on the two surfaces should be identical. A similar behavior for $\mathrm{Si}(111)$ homoepitaxial growth is also reported in Ref. 28. However, at least one group (see Ref. 13) reports a slower transition to amorphous growth on $\mathrm{Si}(111)$ at growth temperatures between 250 and $400{ }^{\circ} \mathrm{C}$. We attribute this difference to discrepancies in the absolute growth temperatures at which these experiments were performed.

In summary, this model concludes that the mechanisms responsible for the formation of amorphous $\mathrm{Si}$ on $\mathrm{Si}(100)$ can be reduced to the same mechanisms responsible for the formation of amorphous $\mathrm{Si}$ on $\mathrm{Si}(111)$. Furthermore, it is speculated that LTE on any $\operatorname{Si}\{h k l\}$ surface can be explained by the formation of critically sized $\mathrm{Si}(111)$ facets and by the mechanisms responsible for the formation of amorphous $\mathrm{Si}$ on $\mathrm{Si}(111)$.

\section{SUMMARY}

In this study, RHEED was used to investigate surface roughening during low temperature $\mathrm{Si}(100)$ homoepitaxy. In order to probe surface roughness and in-plane coherence lengths, RHEED patterns were interpreted using a simple kinematic diffraction model. After calibrating the RHEED 
analyses against TEM analyses of surface roughening, RHEED could be used to accurately estimate average surface roughness during growth.

The RHEED analyses of surface roughening during low temperature $\mathrm{Si}(100)$ homoepitaxy revealed that initially surface roughness scaled linearly with deposited thickness at a rate that decreased with increasing growth temperature. Similarly, the decay of in-plane coherence lengths depended on growth temperature. These temperature dependent roughening behaviors were attributed to barriers to adatom diffusion on the growth surface which could be enhanced by the presence of impurities or step edge diffusion barriers. After the initial linear increase in surface roughness with deposited thickness, the rate of surface roughening decreased and eventually reached a saturation value. This saturation roughness followed an Arrhenius relation with growth temperature, with an activation energy, $E_{\text {act }} \sim 0.31 \mathrm{eV}$, that agreed well with the activation energy for LTE, $E_{\text {act }} \sim 0.35 \mathrm{eV}$. In addition, the formation of facets and twins at the growth surface coincided with the decrease in the rate of surface roughening. Since the final measured coherence lengths near the crystalline/amorphous phase transition also increased with growth temperature, the saturation roughness and in-plane coherence lengths were identified as critical length scales associated with the formation of a critically sized $\operatorname{Si}\{111\}$ facets near $h_{\text {epi }}$. All of these results were incorporated into a model for LTE which links surface roughening to the formation of critically sized $\mathrm{Si}\{111\}$ facets and the eventual breakdown in crystalline growth. It was also suggested that the mechanism(s) for formation of amorphous Si during $\mathrm{Si}(100)$ homoepitaxy can be understood by identifying the mechanism(s) responsible for amorphous phase formation during $\mathrm{Si}(111)$ homoepitaxy.

\section{ACKNOWLEDGMENTS}

The authors would like to thank Eric Chason and Darryl Barlett for stimulating discussions and useful suggestions. This work was supported by the NSF, Contract No. DMR9202176.
${ }^{1}$ V. Drouot, M. Gendry, C. Santinelli, P. Viktorovitch, G. Hollinger, S. Elleuh, and J.-L. Pelouard, J. Appl. Phys. 77, 1810 (1995).

${ }^{2}$ M. Morita, A. Teramoto, K. Makihara, T. Ohmi, Y. Nakazato, A. Uchiyama, and T. Abe, Proceeding of the Third International Symposium on Ultra Large Scale Integration Science and Technology (Electrochemical Society, Pennington, NJ, 1991).

${ }^{3}$ See for example, S. P. Murarka, in Metallization: Theory and Practise for VLSI and USLI (Butterworth and Heinemann, Boston, 1993).

${ }^{4}$ E. Chason, T. M. Mayer, B. K. Kellerman, D. T. McIlroy, and A. J. Howard, Phys. Rev. Lett. 72, 3040 (1994).

${ }^{5}$ C. Orme, M. D. Johnson, J. L. Sudijono, K. T. Leung, and B. G. Orr, Appl. Phys. Lett. 64, 860 (1994).

${ }^{6}$ J. Chevrier, V. LeThanh, R. Buys, and J. Derrien, Europhys. Lett. 16, 737 (1991).

${ }^{7}$ M. Kardar, G. Parisi, and Y.-C. Zhang, Phys. Rev. Lett. 56, 889 (1986).

${ }^{8}$ D. P. Adams, S. M. Yalisove, and D.J. Eaglesham, Appl. Phys. Lett. 63, 3571 (1993)

${ }^{9}$ D. P. Adams and S. M. Yalisove, J. Appl. Phys. 76, 5185 (1994).

${ }^{10}$ D. J. Eaglesham, J. Appl. Phys. 77, 3597 (1995).

${ }^{11}$ D. J. Eaglesham, H.-J. Gossman, and M. Cerullo, Phys. Rev. Lett. 65, 1227 (1990).

${ }^{12}$ M. V. Ramana Murty and H. A. Atwater, Phys. Rev. B 49, 8483 (1994).

${ }^{13}$ J. Chevrier, A. Cruz, N. Pinto, I. Berbezier, and J. Derrien, J. Phys. (France) I 4, 1309 (1994).

${ }^{14}$ The contrast of twins and stacking faults obscures the contrast of the Ge marker layers and hinders TEM analysis of surface roughness.

${ }^{15}$ M. C. Tringides and M. G. Lagally, Reflection High Energy Electron Diffraction and Reflection Electron Imaging of Surfaces, edited by P. K. Larsen and P. J. Dobson, Nato Series (Plenum, New York, 1988).

${ }^{16}$ J. M. Van Hove, P. Pukite, P. I. Cohen, and C. S. Lent, J. Vac. Sci. Technol. A 1, 609 (1983).

${ }^{17}$ N. Masud and J. B. Pendry, J. Phys. C 9, 1833 (1976).

${ }^{18}$ A. Ishizaka and Y. Shiraki, J. Electrochem. Soc. 133, 66 (1986).

${ }^{19}$ D. Barlett, C. W. Snyder, B. G. Orr, and R. Clarke, Rev. Sci. Instrum. 62, 1263 (1991)

${ }^{20}$ The kinematic condition is a form of Z-contrast. S. J. Pennycook and D. E. Jesson, Phys. Rev. Lett. 64, 938 (1990).

${ }^{21}$ J. E. Mahan, K. M. Geib, G. Y. Robinson, and R. G. Long, J. Vac. Sci. Technol. A 8, 3692 (1990).

${ }^{22}$ G. Ehrlich and F. G. Hudda, J. Chem. Phys. 44, 1039 (1966).

${ }^{23}$ J. Tersoff, A. W. Deneir van der Gon, and R. M. Tromp, Phys. Rev. Lett. 72, 266 (1994).

${ }^{24}$ B. S. Swartzentruber, Phys. Rev. Lett. 76, 459 (1996).

${ }^{25}$ J. E. Vasek, Z. Zhang, C. T. Salling, and M. G. Lagally, Phys. Rev. B 51, 17207 (1995).

${ }^{26}$ J. J. Boland, Surf. Sci. 244, 1 (1991)

${ }^{27}$ M. Copel and R. M. Tromp, Phys. Rev. Lett. 72, 1236 (1994).

${ }^{28}$ B. E. Weir, B. S. Freer, R. L. Headrick, D. J. Eaglesham, G. H. Gilmer, J. Bevk, and L. C. Feldman, Appl. Phys. Lett. 59, 204 (1991).

${ }^{29}$ D. J. Eaglesham, A. E. White, L. C. Feldman, N. Moriya, and D. C. Jacobson, Phys. Rev. Lett. 70, 1643 (1993). 